\title{
BMJ Open Systematic review of universal school-based resilience interventions targeting adolescent tobacco, alcohol or illicit drug use: review protocol
}

\author{
Rebecca Kate Hodder, ${ }^{1,2,3}$ Megan Freund, ${ }^{1,2,3}$ Luke Wolfenden, ${ }^{2,3}$ \\ Jenny Bowman, ${ }^{2}$ Karen Gillham, ${ }^{1,3}$ Julia Dray, ${ }^{1,2,3}$ John Wiggers ${ }^{1,2,3}$
}

To cite: Hodder RK, Freund $\mathrm{M}$, Wolfenden $\mathrm{L}$, et al. Systematic review of universal school-based resilience interventions targeting adolescent tobacco, alcohol or illicit drug use: review protocol. BMJ Open 2014;4:e004718. doi:10.1136/bmjopen-2013004718

- Prepublication history and additional material is available. To view please visit the journal (http://dx.doi.org/ 10.1136/bmjopen-2013004718).

Received 19 December 2013 Revised 23 April 2014 Accepted 25 April 2014

CrossMark

For numbered affiliations see end of article.

Correspondence to Rebecca Kate Hodder; Rebecca.hodder@hnehealth. nsw.gov.au

\section{ABSTRACT}

Introduction: Tobacco, alcohol and illicit drug use contribute significantly to global rates of morbidity and mortality. Despite evidence suggesting interventions designed to increase adolescent resilience may represent a means of reducing adolescent substance use, and schools providing a key opportunity to implement such interventions, existing systematic reviews assessing the effectiveness of school-based interventions targeting adolescent substance use have not examined this potential.

Methods and analysis: The aim of the systematic review is to determine whether universal interventions focused on enhancing the resilience of adolescents are effective in reducing adolescent substance use. Eligible studies will: include participants $5-18$ years of age; report tobacco use, alcohol consumption or illicit drug use as outcomes; and implement a school-based intervention designed to promote internal (eg, selfesteem) and external (eg, school connectedness) resilience factors. Eligible study designs include randomised controlled trials, cluster randomised controlled trials, staggered enrolment trials, stepped wedged trials, quasi-randomised trials, quasiexperimental trials, time series/interrupted time-series trials, preference trials, regression discontinuity trials and natural experiment studies with a parallel control group. A search strategy including criteria for participants, study design, outcome, setting and intervention will be implemented in various electronic databases and information sources. Two reviewers will independently screen studies to assess eligibility, as well as extract data from, and assess risk of bias of included studies. A third reviewer will resolve any discrepancies. Attempts will be made to quantify trial effects by meta-analysis. Binary outcomes will be pooled and effect size reported using ORs. For continuous data, effect size of trials will be reported using a mean difference where trial outcomes report the same outcome using a consistent measure, or standardised mean difference where trials report a comparable measure. Otherwise, trial outcomes will be described narratively.

Dissemination: Review findings will be disseminated via peer-reviewed journals and conferences.

\section{INTRODUCTION}

Tobacco, alcohol and illicit drug use contribute significantly to global rates of morbidity and mortality. ${ }^{2}$ School-based interventions have been recommended to be implemented to reduce this burden given initiation of such drug use typically occurs during adolescence, ${ }^{3}$ and schools provide almost universal access to adolescents for prolonged periods. Given this, school-based interventions have been implemented by governments internationally in an attempt to reduce adolescent initiation to substance use. ${ }^{4-6}$

Despite widespread implementation, Cochrane reviews have found little evidence for the effectiveness of school-based drug prevention programmes on adolescent substance use, with such reviews focused on any or only universal intervention approaches. ${ }^{4-6}$ Of the multiple intervention approaches examined by such reviews, little or no evidence of effectiveness has been found for the most commonly implemented curricula or information-only interventions. Some evidence, however, has been found for various psychosocial interventions, including those that adopt a social competence and social influence, generic psychosocial or individual social skills approach. ${ }^{4-6}$ A review by the WHO examining school-based drug prevention programmes similarly concluded that programmes that promote young people's mental well-being were most likely to be effective, suggesting that interventions incorporating a mental wellbeing approach may have the best chance of impacting substance use. ${ }^{7}$

The concept of resilience and closely related research regarding protective factors provides one avenue for addressing mental well-being that is suggested to have an impact on adolescent substance use..$^{8-17}$ Resilience has been variably defined as the 
process of, capacity for, or outcome of successful adapta-

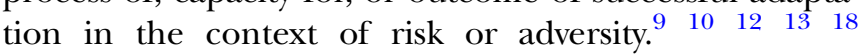
Despite this variability, it is generally agreed that a range of individual and environmental protective factors are thought to: contribute to an individual's resilience; be critical for positive youth development and protect adolescents from engaging in risk behaviours, such as substance use. ${ }^{19-22}$ Individual or internal resilience factors refer to the personal skills and traits of young people (including self-esteem, empathy and self-awareness). ${ }^{23}$ Environmental or external resilience factors refer to the positive influences within a young person's social environment (including connectedness to family, school and community). ${ }^{23}$ Various studies have separately reported such factors to be negatively associated with adolescent use of different types of substances, ${ }^{12} \quad 16 \quad 24-36$ for example, higher self-esteem ${ }^{162932} 35$ is associated with lower likelihood of tobacco and alcohol use.

Despite this associative evidence, to the authors' knowledge, existing systematic reviews assessing the effectiveness of school-based substance use interventions have not reported the effectiveness of universal resilience-based interventions on adolescent substance use. $^{4-637}$ Three existing Cochrane reviews have individually examined the efficacy of school-based tobacco, alcohol and illicit drug use programmes. ${ }^{4-6}$ Such reviews have not reported outcomes for universal resiliencebased interventions specifically, but have included such interventions in broader categories of intervention type for subgroup analysis. As a consequence, a systematic review of the efficacy of universal resilience-based interventions specifically remains unreported. For example, a tobacco-focused review which included any intervention type, classified interventions with a component of resilience content into different subgroups such as social competence or social influence interventions, finding evidence for both broad intervention approaches. ${ }^{6}$ For the alcohol-focused review, only universal interventions were included with such interventions grouped according to whether they targeted alcohol alone or targeted multiple substance types. ${ }^{5}$ While meta-analysis was not conducted due to the heterogeneity of studies, the review concluded that some psychosocial and developmental prevention programmes were effective. Given such inability to draw conclusions with respect to universal resilience interventions and studies suggesting an association exists between resilience and substance use, there is a need to examine whether more specifically defined universal resilience interventions are efficacious in reducing substance use by adolescents. Such a review has the potential to identify studies not included in existing Cochrane reviews which, at the time of publication, were conducted up to 10 years ago.

\section{Objective}

To determine whether universal school-based interventions designed to enhance resilience are efficacious relative to a comparison group in reducing the extent of adolescent tobacco, alcohol or illicit drug use.

\section{METHODS}

All methods employed in the review will be consistent with the Cochrane Handbook for Systematic Reviews of Interventions. $^{38}$

\section{Eligibility criteria \\ Study characteristics \\ Participants}

Studies will be included if they report results of participants aged 5-18 years. Studies that select participants based on a diagnosis of psychiatric or other mental illness, cognitive or developmental disability will be excluded from the study. There will be no exclusions on the basis of study country.

\section{Study design}

Studies with the following designs will be included: randomised controlled trials including cluster randomised controlled trials; staggered enrolment trials ${ }^{39}$ or stepped wedged trials $^{40}$; quasi-randomised trials where group allocation is not purely random ${ }^{41}{ }^{42}$; quasi-experimental trials including non-randomised pre-post ${ }^{43}$; time series/ interrupted time-series trials including multiple baseline trials with independent control groups ${ }^{39} 43$; preference trials $^{40}$ and regression discontinuity trials ${ }^{39}$ and natural experiment studies. ${ }^{44}$ Trials with non-random assignment of groups will be included given Medical Research Council recommendations that non-randomised designs may represent the most appropriate evaluation deign for some complex public health interventions, ${ }^{45}$ and as an acknowledgment of the value of non-randomised designs in assessing intervention effects in public health interventions. ${ }^{46}$ Studies with a length of follow-up of at least 6 months post intervention start will be included in the review. Studies will be excluded if they do not include a parallel comparison group.

\section{Comparison group}

Studies with a comparison group that received no intervention, usual practice, attention only or an alternate intervention will be included.

\section{Primary outcomes}

Studies will be included if they report one or more of the following outcomes:

- Tobacco use (including but not limited to proportion ever smoked, frequency of smoking, number of cigarettes smoked, tobacco use in the past week, current smoking status or established tobacco use);

- Alcohol consumption (including but not limited to proportion ever consumed an alcoholic drink, alcohol use in the past week, frequency of alcohol consumption, binge drinking or established alcohol use); 
- Illicit drug use (including but not limited to ever use or frequency of use of any illicit drug or a specific drug eg, cannabis, amphetamines or cocaine).

Substance-use data collected via various methods will be included, for example, data collected via observation; self-report via face to face or telephone, internet survey; secondary report by peers or parents; and biochemical measurement of substance use (such as carbon monoxide or cotinine detection).

\section{Secondary outcomes}

Any adverse outcomes reported in included studies will be described in the results.

\section{Interventions}

A study will be included if it reports a universal intervention that specifically aims to improve at least one internal and at least one external resilience factor. A universal intervention is defined as an intervention delivered to an entire school population. As the internal and external factors that comprise resilience are not consistently reported, numerous bodies of work were reviewed to identify an inclusive list of internal and external resilience factors. ${ }^{9} 10121318$ Internal resilience factors will include: cooperation and communication, self-efficacy, self-esteem, empathy, problem solving, decision-making skills, autonomy, self-awareness, goals and aspirations, social and emotional skills or competence and selfcontrol or self-regulation. ${ }^{9} 1012131847-49$ External resilience factors will include: meaningful participation, high adult expectations, caring relationships and support within home, school and community environments; peercaring relationships and prosocial peers. ${ }^{9} 10121847-49$

Given the theoretical and componentry crossover between resilience and other intervention approaches (such as strengths based, social competence, social influence, skills focused, affective focused, social and emotional learning/well-being, mental well-being and psychosocial $^{50-53}$ ), a study will be included irrespective of the stated overall intervention approach if it specifically aims to address at least one internal and one external resilience factor as defined above. Studies will be included irrespective of whether substance use is the primary outcome measure.

There will be no exclusion criteria regarding other intervention elements, the duration of intervention, the format of intervention delivery (eg, curricula based or internet based) or the intervention administration (eg, the intervention could be delivered by school staff, research staff, community members or students).

\section{Setting}

Studies will be included if the intervention is implemented across a whole school.

\section{Publication characteristics}

Studies of any language will be included and translated using Google translate where required. Studies published in the past 20 years in peer-reviewed journals will be eligible for inclusion.

\section{Information sources \\ Electronic databases}

The following electronic databases will be searched: Cochrane Central Register of Controlled Trials, MEDLINE, EMBASE, CINAHL, PsycINFO, ERIC and the first 200 citations only of Google Scholar.

\section{Other sources}

The following additional information sources will be searched or contacted for eligible studies:

- Hand searching of three relevant journals in the field (past 5 years; Addiction, Journal of Adolescent Health, Journal of School Health);

- Reference lists of included studies;

- Reference lists of existing Cochrane reviews on school-based interventions targeting tobacco, alcohol and illicit substances ${ }^{4-6}$;

- Corresponding authors of included studies;

- PubMed single citation searcher.

\section{Search strategy}

The search strategy will include terms for participants, setting, intervention, study design ${ }^{54}$ and outcome (sourced from current Cochrane systematic reviews examining the effectiveness of tobacco, alcohol and illicit substance use interventions; see web only appendix 1 for MEDLINE search strategy). ${ }^{4-6}$ The search strategy will be tailored as required for implementation in other information sources.

\section{Study selection}

Two reviewers will independently screen the titles and abstracts of all studies identified via the implementation of the above search strategy. The reviewers will not be blind to study authors. A standardised screening tool will be used to assess study eligibility with those titles and abstracts not meeting the criteria excluded from the review (see web only appendix 2). The full texts of the remaining papers will be sourced and examined independently by the two reviewers to assess study eligibility. Any disagreement between the two reviewers regarding study eligibility that cannot be resolved via consensus will be assessed by a third reviewer. Corresponding authors will be contacted if there is not sufficient information to determine eligibility. If sufficient information remains unavailable, the study will be deemed ineligible. The details of ineligible studies for which the full text was sourced will be reported in the Results section including the reason the study was ineligible.

\section{Data extraction}

The two study reviewers will independently extract data from the eligible studies using a standardised form. Reviewers will not be blind to study authors. Any unresolved discrepancies between reviewers regarding the 
extracted data will be resolved by the third reviewer. Where there is insufficient data to make a judgement regarding eligibility, the corresponding authors will be contacted for clarification.

The following information will be extracted from eligible studies where available: authors, year of publication, year/s of study, country, study design, intervention (including resilience factors targeted, duration, intensity), comparison group type, substance use targeted, measurement tool characteristics, study participants' demographics (including age and gender), study results (including sample size, consent rate/s, participation rate/s, length of follow-up, attrition, relevant outcome results and intraclass correlation), intervention fidelity (including any process measures) and information to determine any potential study bias (see below).

\section{Assessment of risk of bias}

Study bias of eligible studies will be assessed independently by the two reviewers against the Cochrane Handbook for Systematic Reviews of Interventions study characteristics including: sequence generation (selection bias), allocation sequence concealment (selection bias), blinding of participants and personnel (performance bias), blinding of outcome assessment (detection bias), incomplete outcome data (attrition bias), selective outcome reporting (reporting bias) and other potential sources of bias. ${ }^{38}$

Included non-RCTs will be assessed for selection bias that may have resulted in confounding of the outcome of interest using the Newcastle-Ottawa Scale, ${ }^{38}$ and where possible statistical methods will adjust for such confounding. Any additional biases specific to individual study designs will be assessed by the reviewers and reported. ${ }^{38}$

The reviewers will not be blinded to the names of the authors, institutions, journal or results of studies. Any disagreement between the two reviewers regarding study bias that is not resolved via discussion will be resolved by a third reviewer.

\section{Data analysis}

\section{Data synthesis and analysis}

Attempts will be made to quantify trial effects from randomised controlled trials by meta-analysis using data from intention-to-treat analyses. Where multiple measures (eg, biochemical and self-reported smoking status) for the same outcome are reported, the most objective measure of outcome will be used. Similarly, where studies report data from multiple follow-up periods, data from final follow-up periods will be extracted. Binary outcomes (such as tobacco use) will be pooled and effect size reported using ORs. For continuous data, the effect size of trials will be reported using a mean difference where trial outcomes are reported using a consistent measure, or a standardised mean difference where outcomes across trials report the same outcome using comparable measures. Sensitivity analysis will be conducted excluding trials judged to be at high risk of bias. Meta-analyses will be performed using a random effects model, when there is little evidence of heterogeneity $\left(\mathrm{I}^{2}<50 \%\right)$ and only for randomised trials. Otherwise trial outcomes, including those from non-randomised trials, will be described narratively.

\section{Assessment of study heterogeneity}

Study heterogeneity will be assessed via examination of forest plots and calculation of $\mathrm{I}^{2}$ statistic. If an $\mathrm{I}^{2}$ score over $50 \%$ is found, the cause of the heterogeneity will be explored via the conduct of subgroup analyses and sensitivity analysis via meta-regression.

\section{Issues of clustering}

If any included cluster randomised controlled trials have not accounted for clustering, intraclass correlations will be requested from authors or if not available, estimates from similar studies (defined as those with similar school and student characteristics including gender and scholastic year proportions) will be used to adjust for clustering.

\section{Dealing with missing data}

Authors of included studies will be contacted to provide any missing data (eg, missing participant data due to drop out or missing statistics such as SDs). If not available, attempts will be made to compute them, including an intention-to-treat analysis where appropriate.

\section{Assessment of reporting bias}

Possible reporting bias will be determined by examining funnel plots of the included studies and comparison with trial registers.

\section{Additional analyses}

If possible, additional analyses will be conducted by subgroup (eg, gender), intervention intensity, intervention duration and length of follow-up. Further subgroup analysis is planned based on whether included interventions focused solely on resilience (resilience interventions) versus interventions that focused on resilience as well as other determinants of substance use (multidimensional interventions).

\section{ETHICS AND DISSEMINATION}

Given this is a systematic review, ethics approval is not required. Findings of this review will be disseminated via peer-reviewed journals and conference presentations.

\section{DISCUSSION}

This systematic review will be the first internationally to examine the effectiveness of universal school-based resilience interventions in reducing the prevalence of adolescent tobacco, alcohol and illicit drug use. Given that the majority of adolescents attend school, population-level implementation of an effective 
intervention approach has the potential to provide significant health gains by reducing adolescent substance use, and as a result will be of interest to researchers and policy makers.

\section{Author affiliations}

${ }^{1}$ Hunter New England Population Health, Hunter New England Local Health District, Wallsend, New South Wales, Australia

${ }^{2}$ The University of Newcastle, University Drive, Callaghan, New South Wales, Australia

${ }^{3}$ Hunter Medical Research Institute, New Lambton, New South Wales, Australia

Contributors RKH led the drafting of the protocol and will lead the review. All the authors contributed to the refinement of the review protocol, approved the final manuscript and will be involved in the preparation of the review.

Competing interests None.

Provenance and peer review Not commissioned; externally peer reviewed.

Open Access This is an Open Access article distributed in accordance with the Creative Commons Attribution Non Commercial (CC BY-NC 3.0) license, which permits others to distribute, remix, adapt, build upon this work noncommercially, and license their derivative works on different terms, provided the original work is properly cited and the use is non-commercial. See: http:// creativecommons.org/licenses/by-nc/3.0/

\section{REFERENCES}

1. Begg S, Vos T, Barker B, et al. The burden of disease and injury in Australia 2003. PHE 82, Canberra: AlHW, 2007.

2. Lopez AD. Global burden of disease and risk factors. Washington DC: Oxford University Press, 2006.

3. Gilman SE, Abrams DB, Buka SL. Socioeconomic status over the life course and stages of cigarette use: initiation, regular use, and cessation. J Epidemiol Community Health 2003;57:802-8.

4. Faggiano F, Vigna-Taglianti FD, Versino E, et al. School-based prevention for illicit drugs' use. Cochrane Database Syst Rev 2005; (2):CD003020.

5. Foxcroft DR, Tsertsvadze A. Universal school-based prevention programs for alcohol misuse in young people. Cochrane Database Syst Rev 2011;5:CD009113.

6. Thomas RE, McLellan J, Perera R. School-based programmes for preventing smoking. Cochrane Database Syst Rev 2013(4): CD001293.

7. Stewart-Brown S. What is the evidence on school health promotion in improving health or preventing disease and, specifically, what is the effectiveness of the health promoting schools approach? Copenhagen, WHO Regional Office for Europe (Health Evidence Network report), 2006.

8. Resnick MD. Resilience and protective factors in the lives of adolescents. J Adolesc Health 2000;27:1-2.

9. Masten AS. Ordinary magic. Resilience processes in development Am Psychol 2001;56:227-38.

10. Luthar SS, Cicchetti D, Becker B. The construct of resilience: a critical evaluation and guidelines for future work. Child Dev 2000;71:543-62.

11. Greenberg MT, Weissberg RP, O'Brien MU, et al. Enhancing school-based prevention and youth development through coordinated social, emotional, and academic learning. Am Psycho 2003;58:466-74.

12. Fergus S, Zimmerman MA. Adolescent resilience: a framework for understanding healthy development in the face of risk. Annu Rev Public Health 2005;26:399-419.

13. Harvey J, Delfabbro PH. Psychological resilience in disadvantaged youth: a critical overview. Aust Psychol 2004;39:3-13.

14. Catalano RF, Berglund ML, Ryan JAM, et al. Positive Youth Development in the United States: Research Findings on Evaluations of Positive Youth Development Programs. Ann Am Acad Pol Soc Sci 2004;591:124.

15. Greenberg MT. Promoting resilience in children and youth: preventive interventions and their interface with neuroscience. Ann N Y Acad Sci 2006;1094:139-50.
16. Resnick MD, Bearman PS, Blum RW, et al. Protecting adolescents from harm. Findings from the National Longitudinal Study on Adolescent Health. JAMA 1997;278:823-32.

17. Resnick MD. Protective factors, resiliency and healthy youth development. Adolesc Med: State Art Rev 2000;11:157-64.

18. Lee TY, Cheung CK, Kwong WM. Resilience as a positive youth development construct: a conceptual review. Scientific World Journal 2012;2012:390450.

19. Benard B. Fostering Resiliency in Urban Schools. Closing the achievement gap: a vision for changing beliefs and practices. Virginia: Association for Supervision and Curriculum Development, 1996.

20. Benard B. Protective factors in the family, school, and community. Portland, OR: Western Center for Drug-Free Schools and Communities, 1991.

21. Bernat DH, Resnick MD. Healthy youth development: science and strategies. J Public Health Manag Pract 2006(Suppl):S10-16

22. Toumbourou JW. Drug prevention strategies: a developmental settings approach prevention research evaluation report Number 2. Melbourne, Australian Drug Foundation, 2002.

23. MindMatters Evaluation Consortium. Report of the MindMatters (National Mental Health in Schools Project) Evaluation Project, vols. 1-4. Newcastle: Hunter Institute of Mental Health, 2000.

24. Bond L, Butler $\mathrm{H}$, Thomas L, et al. Social and school connectedness in early secondary school as predictors of late teenage substance use, mental health, and academic outcomes. J Adolesc Health 2007;40:357.e9-18.

25. Carvajal SC, Granillo TM. A prospective test of distal and proximal determinants of smoking initiation in early adolescents. Addict Behav 2006;31:649-60.

26. Fang L, Barnes-Ceeney K, Schinke SP. Substance use behavior among early-adolescent Asian American girls: the impact of psychological and family factors. Women Health 2011;51:623-42.

27. Haegerich TM, Tolan PH. Core competencies and the prevention of adolescent substance use. New Dir Child Adolesc Dev 2008:2008:47-60.

28. Hiemstra M, Otten R, Engels RC. Smoking onset and the time-varying effects of self-efficacy, environmental smoking, and smoking-specific parenting by using discrete-time survival analysis. $J$ Behav Med 2012;35:240-51.

29. Kavas AB. Self-esteem and health-risk behaviors among Turkish late adolescents. Adolescence 2009;44:187-98.

30. McLellan L, Rissel C, Donnelly N, et al. Health behaviour and the school environment in New South Wales, Australia. Soc Sci Med 1999;49:611-19.

31. Neumark-Sztainer D, Story M, French SA, et al. Psychosocial correlates of health compromising behaviors among adolescents. Health Educ Res 1997;12:37-52.

32. Patton GC, Bond L, Carlin JB, et al. Promoting social inclusion in schools: a group-randomized trial of effects on student health risk behavior and well-being. Am J Public Health 2006;96:1582-7.

33. Piko BF, Kovacs E. Do parents and school matter? Protective factors for adolescent substance use. Addict Behav 2010;35:53-6.

34. Vuille JC, Schenkel M. Social equalization in the health of youth The role of the school. Eur J Public Health 2001;11:287-93.

35. Wiefferink $\mathrm{CH}$, Peters L, Hoekstra F, et al. Clustering of health-related behaviors and their determinants: possible consequences for school health interventions. Prev Sci 2006;7:127-49.

36. Wright LL, Palfai TP. Life goal appraisal and marijuana use among college students. Addict Behav 2012;37:797-802.

37. Thomas R, Perera R. School-based programmes for preventing smoking Cochrane Database Syst Rev 2006:3.CD001293.

38. Higgins JPT, Green S eds. Cochrane handbook for systematic reviews of interventions. Version 5.1.0 [updated March 2011]. The Cochrane Collaboration, 2011.

39. Mercer SL, DeVinney BJ, Fine LJ, et al. Study designs for effectiveness and translation research: identifying trade-offs. $A m J$ Prev Med 2007;33:139-54.

40. Craig $\mathrm{P}$, Dieppe $\mathrm{P}$, Macintyre $\mathrm{S}$, et al. Developing and evaluating complex interventions: the new Medical Research Council guidance. BMJ 2008;337:a1655.

41. Battaglia MP, Link MW, Frankel MR, et al. An evaluation of respondent selection methods of respondent mail surveys. Public Opin Q 2008;72:459-69.

42. Oldendick RW, Bishop GF, Sorenson SB, et al. A comparison of the Kish and last birthday methods of respondent selection in telephone surveys. J Off Stat 1988;4:307-18.

43. Nutbeam D, Bauman A. Evaluation in a nutshell: a practice guide to the evaluation of health promotion programs. North Ryde, Australia: McGraw-Hill, 2006. 
44. Sadish W, Cook T, Campbell D. Experimental and quasi-experimental designs for generalized causal inference. New York: Houghton Mifflin Company, 2002.

45. Craig P, Dieppe P, Macintrye S, et al. Developing and evaluating complex interventions: new guidance. Medical Research Council, 2008.

46. Armstrong R, Waters E, Jackson N, et al. Guidelines for systematic reviews of health promotion and public health interventions. [Version 2]. Australia: Melbourne University, 2007.

47. Measuring protective factors and resilience traits in youth: The healthy kids resilience assessment. Seventh annual meeting of the society for prevention research. California: School and Community Health Research Group, 1999.

48. Hanson TL, Kim JO. Measuring resilience and youth development: the psychometric properties of the Healthy Kids Survey. Washington, DC, USA: Department of Education, Institute of Education Sciences, National Centre for Education Evaluation and Regional Assistance, Regional Educational Laboratory West, 2007.
49. Masten AS. Resilience in developing systems: progress and promise as the fourth wave rises. Dev Psychopathol 2007;(3):921-30.

50. Brownlee K, Rawana J, Franks J, et al. A systematic review of strengths and resilience outcome literature relevant to children and adolescents. Child Adolesce Soc Work J 2013;30:435-59.

51. Griffin KW, Botvin GJ. Evidence-based interventions for preventing substance use disorders in adolescents. Child Adolesc Psychiatr Clin North Am 2010;19:505-26.

52. Hawkins JD, Catalano RF, Miller JY. Risk and protective factors for alcohol and other drug problems in adolescence and early adulthood: implications for substance abuse prevention. Psychol Bull 1992;112:64-105.

53. Petraitis J, Flay BR, Miller TQ. Reviewing theories of adolescent substance use: organizing pieces in the puzzle. Psychol Bull 1995;117:67-86.

54. Kingsland $\mathrm{M}$, Wiggers $\mathrm{J}$, Wolfenden $\mathrm{L}$. Interventions in sports settings to reduce alcohol consumption and alcohol-related harm: a systematic review. BMJ Open 2012;2:e000645, 1-6. 